\title{
Research on optical flat models of stresses in the crankpin of internal combustion engine
}

\author{
Olga Zhed ${ }^{1, *}$, Vladimir Kopylov ${ }^{1}$, and Adolf Koshelenko ${ }^{1}$ \\ ${ }^{1}$ Peoples Friendship University of Russia (RUDN University), 6 Miklukho-Maklaya St, Moscow, \\ 117198, Russian Federation
}

\begin{abstract}
The intensity of the contour strains of the oil channel is determined by the method of photomechanics in the consequence of the loads of the crankpin in the working cycle of the internal combustion engine. The studies have made it possible to establish the qualitative and quantitative composition of the stresses on the hole contour of the oil channel against deformation of the bending and torsion of its connecting rod.
\end{abstract}

\section{Introduction}

In this paper, we study the distribution of stresses in the flat model of the crankshaft by the method of photomechanics [1] with the aim of improving its design and calculating methodology. Cranked shafts are destroyed by fatigue in places with the greatest concentration of stresses. Such areas are the outlets of the opening for the lubricant material on the root and crankpins of the shaft and in fillets in the mattings of the necks with the cheeks of the shaft (Fig. 1). The models of shafts are loaded according to two schemes (Fig. 2): bending in the flatness of the crank $M_{\text {bend }}$ and torsion $M_{\text {tors }}$.

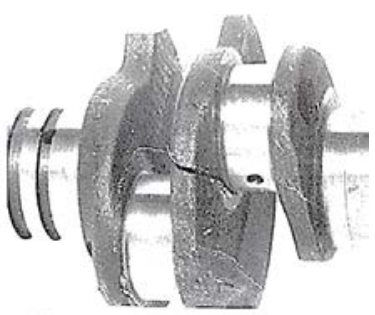

Fig. 1. A photo of a fragment of a cranked shaft with a crack, which was born in a transitional fillet from the connecting rod to the cheek

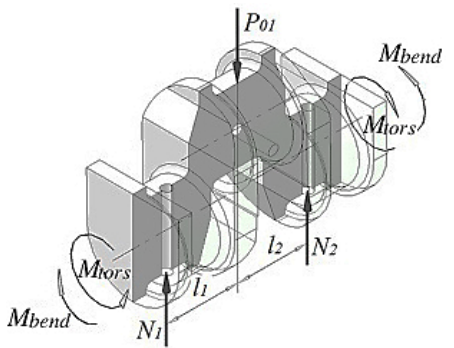

Fig. 2. The scheme of the crankshaft loading: $M_{\text {bend }}$ - bending;

$M_{\text {tors }}$ - torsion;

$N_{1}, N_{2}$ - reactions of the supports;

$P_{01}$ - loading transverse force

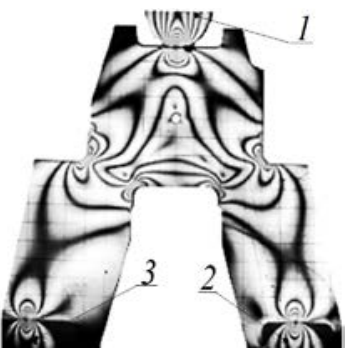

Fig. 3. Photogram of the crankpin model with the oil channel hole: $1-3$ - the optical disk dynamometers

\footnotetext{
"Corresponding author: zhed_ov@rudn.university
} 
The crankpin is calculated only for torsion, and the connecting rod is for bending and torsion. The calculated points are the edge of the grease hole and the fillet of crankpin. In this research, we study the distribution of stresses from bending and torsion along the contour of the hole for supplying lubricant to the crankpin $[4,5]$. In physical experiments, flat models of the crankshaft were used from the optically sensitive material E2, which were investigated in papers $[2,3]$.

\section{Calibration of the model material and methods of photo- optical experiment}

The holes of the oil channels $d=0.6 \mathrm{~cm}$ were drilled in the crankpins (Fig. 2, 3). The crankshafts on the optical disk dynamometers 3 and 2, which fix the reactions of the support $N 1, N 2$ of the crankpins at a distance $l_{1}, l_{2}$, respectively, from the loading transverse force $P_{01}$ applied in the middle of the crankpin through optical disk dynamometer 1, support the crankshaft model. From the calibration of the model material on disks of diameter $d=4 \mathrm{~cm}$, the force for one strip in the center of the disk is $P_{01}=215.5 \mathrm{~N}$.

The studied region of the model under study is the outline of the oil channel hole. For the case when the axis of the opening of the oil channel is perpendicular to the plane of action of the shear force, the bending moment does not cause concentration of the stresses of the contour of the hole (Fig. 3 - 4, b). It is located in the isotropic zone of the crankpin, where there are no tangential stresses. We compare photograms of crankpins, which were obtained during the physical experiment. We are convinced that along the contour of the hole there are areas with zero level of the maximum tangential stresses (Fig. 4, b). The hole of the oil channel didn't influence in the transition fillets from the cheeks to the crankpins.

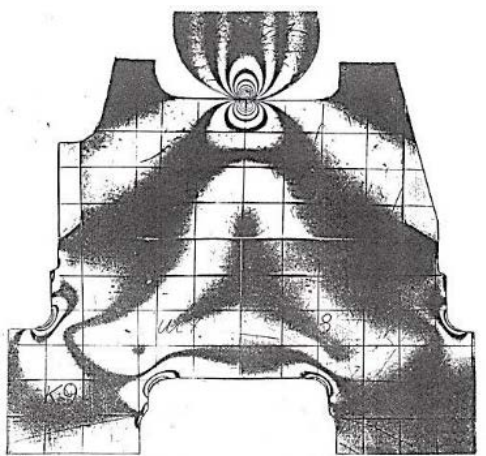

a

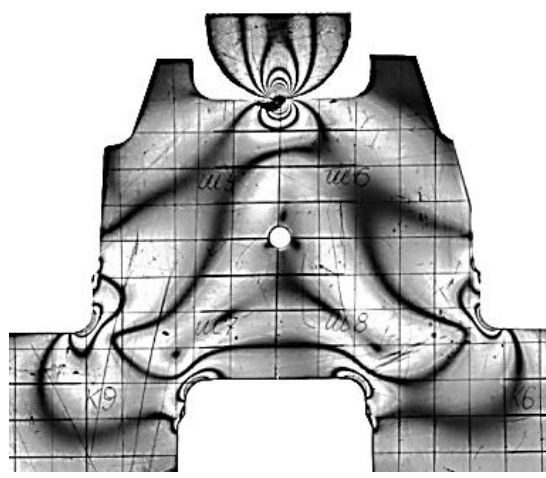

b

Fig. 4. Photograms in the monochromatic light of a mercury lamp compared variants of equally loaded crankpins of flat models of a cranked shaft: a - neck without a hole in the oil channel; b - neck with a hole in the oil channel

Otherwise, the contour of the hole of the oil channel is deformed if its axis is located in the plane of action of the bending moment. During the rotation of the crankshaft by one turn, the longitudinal axis of the oil channel hole is located two times in the plane of action of the bending moment. On the side of the shearing force application, there is a deformation of the compression of the outer contour of the crankpin, and on the opposite side of it, there is a deformation of stretching. In connection with this, the contour of the hole of the oil channel will be subjected to similar deformations. In the photograph Fig. 3, it can be seen that on the side of the stretched fibers of the outer contour in the middle section of the 
crankpin along the line of action of the shearing force $P_{01}$, the deformation strain is $n=2.8$ bands.

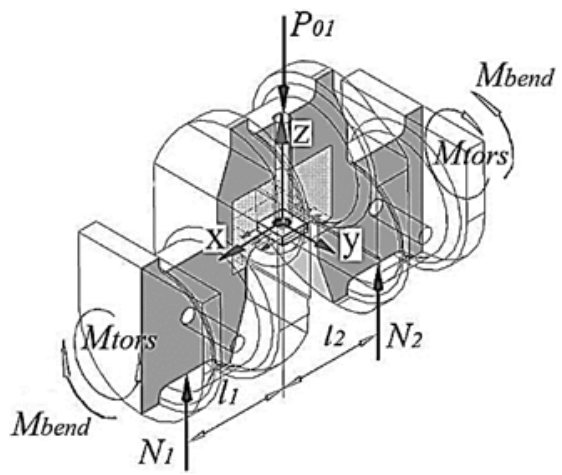

Fig. 5. Scheme of the selection of the plastic model from the 3D image of the connecting rod of the crankpin: $M_{\text {bend }}$ - bending; $M_{\text {tors }}$ - torsion; $N_{1}, N_{2}$ - reactions of the supports $P_{01}$ - loading transverse force

However, if in the middle section on the crankpin contour there is a hole of the oil channel, then the stressed state of this zone receives an additional concentration. The problem of the stressed state of the outline of the hole becomes volumetric and it should be solved by the freezing method on the volumetric model of the cylinder shaft, which is not considered in the present work. A simplified solution of this problem is proposed on the plane straight model, which is made in the form of a plate with a hole, the axis of which is symmetrical to its longitudinal contour.

The geometric parameters of such a plate fit into the three-dimensional model (Fig. 5) of the connecting rod on the side of the stretched fibers with the position of the oil channel hole along the action line of the shearing force. The plate is loaded by the axial force in the longitudinal direction by strain deformation, corresponding to $n=2.8$ bands.

To modulate a uniaxial stretching of a plate with a hole from the bend in the plane of the knee, and then on the same plate of a pure shear, a loading device- stand was developed and made (Fig. 6).

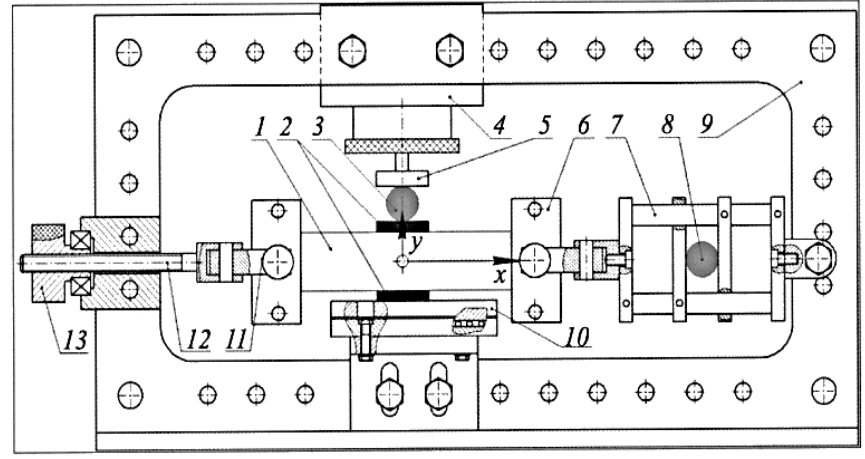

Fig. 6. The device for modeling in a flat straight plate deformations of pure stretching and pure shear on the crankpin:

1 - plate; 2 - support steel bars; 3, 8 - optical dynamometers;

4, 5 - compression force facility; 6, 11 - clamps; 7 - reverser;

9 - frame; 10 - support; 12 - rod; 13 - nut

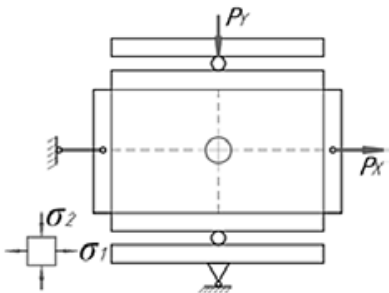

Fig. 7. Modelling on the flat straight plate of the pure shear of the hole contour caused by the crankpin torsion: $P_{x}$ - direction by stretching; $P_{y}$ - direction by compression 
The experiment with stretching of the plate on the stand is resolved to the following actions. The plate 1 of the optically sensitive material $E 2$ is clamped in the clamps 6,11 and mounted in the lever system of the device for loaded model. There is a reverser 7 to the right of the plate that converts the axial tensile force of the plate into the compression of a disk dynamometer 8 that fixes the amount of applied force produced by the rod 12 through the left clamp of the model when screwing the nut 13 .

In the first series of experiments, when the plate is loaded with uniaxial tension, the support steel bars 2 and the optical dynamometer 3 in the loading scheme are absent.

In the second series of experiments, a pure shear is modeled. Model 1 with open-end holes was loaded in the direction $P_{x}$ by stretching and in direction $P_{y}$ - by compression (Fig. 6, 7).

In the first series of experiments, the plate-model was loaded with an axial force in the longitudinal direction along the $\mathrm{x}$-axis by strain expansion corresponding to $n=1.45$ bands. This stress level was recorded on the neck photos (Fig. 4, b) in the median section on its free contour along the action of the cutoff force $P_{01}$, the value of which is determined on the same photogram by the number of bands in the center of the disk dynamometer. According to the calibration of the model material on disks with a diameter $d=4 \mathrm{~cm}$, the force for one strip in the center of the disk is $215.5 \mathrm{~N}$, so for $n=2$ bands in the center of the disk 1 we obtain the shear force $P_{01}=431 \mathrm{~N}$.

In the middle section of the crankpin, regardless of the presence of the oil channel opening, under this load, the same level of the contour stress takes place, which is $n=1.45$ bands. It is this value of the contour stress in the middle section of the crankpin that is used to calculate the loading force of the plate-model with the hole. We transform the order of the strip into the dimension of the normal stresses on the neck contour: $\sigma_{\text {middle }}=n \cdot \sigma_{0}^{t} \cong$ $4 \mathrm{MPa}$, where $\sigma_{0}^{t}=2.76 \mathrm{MPa}$ - the amount of the model band from the calibration. The active cross section of the plane model with allowance for its width $b=2.4 \mathrm{~cm}$ and the thickness of the OSM (optically sensitive material) $t=0.5 \mathrm{~cm}$ was $F=1.2 \mathrm{~cm}^{2}$.

This information makes it possible to calculate the amount of force required to load a plate-model with an aperture, in modeling its extension, by providing an experimentally established value of stresses in the middle section when there is no hole in the neck channel at this location of the neck contour. Let us determine the force with which to stretch the plate-model with the hole: $P_{\text {stretch }}=\sigma_{\text {middle }} \cdot F=480 \mathrm{~N}$. In this case, we load the platemodel with the tensile force $P_{\text {stretch }}=480 \mathrm{~N}$ along the X-axis. The value of this force is given by a disk optical dynamometer 8 (see Fig. 6) with a diameter $d=1.6 \mathrm{~cm}$. The loading scheme of the plate-model by pure stretching and the pure-strain photogram obtained in the polarized light of a mercury lamp are shown in Fig. 8.

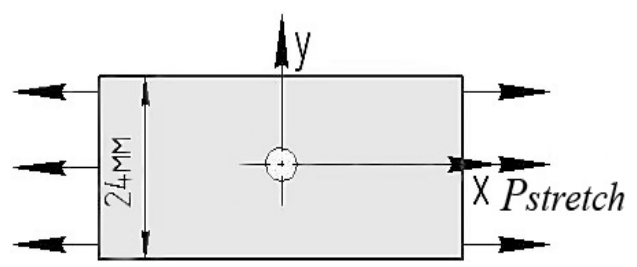

$\mathbf{a}$

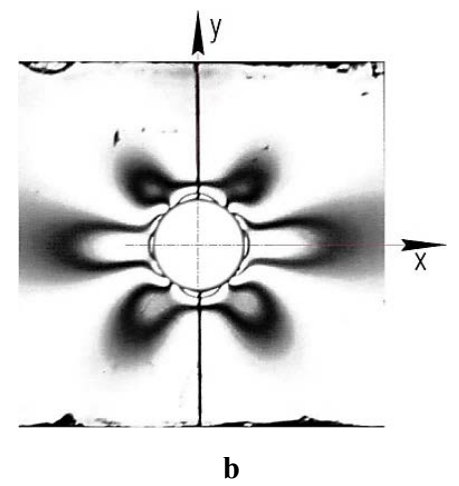

Fig. 8. Loading scheme and photogram of the plate-model: a - pure stretching; $\mathbf{b}$ - photogram of pure stretching, obtained in the polarized light of a mercury lamp 
In the second part of the experiment, the plate-model with the hole was loaded according to the scheme of pure shear (Fig. 9, a). The procedure for calculating the force for loading the model with a pure shear was reduced to determining the torsional moment (Fig. 9, b), created by the sheer force $P_{01}=431 \mathrm{~N}$ and, consequently, the same level of stresses on the neck contour in the middle section 1.45 bands. Taking into account the crank radius $R_{c r}=6.7 \mathrm{~cm}$ and the shear force $P_{01}=431 \mathrm{~N}$, the torque, reduced to the radius of the connecting rod neck $R_{s h}=4.4 \mathrm{~cm}$, is balanced by the force $P_{\text {torsion }}=656 \mathrm{~N}$.

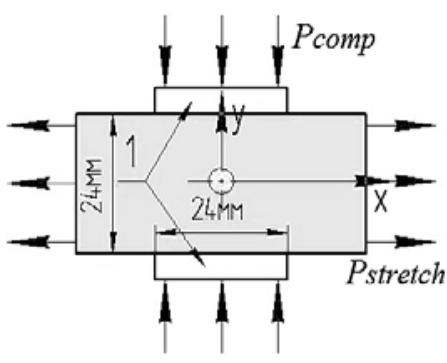

$\mathbf{a}$

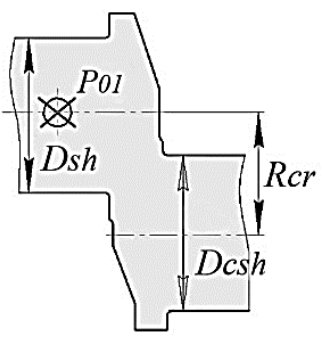

b

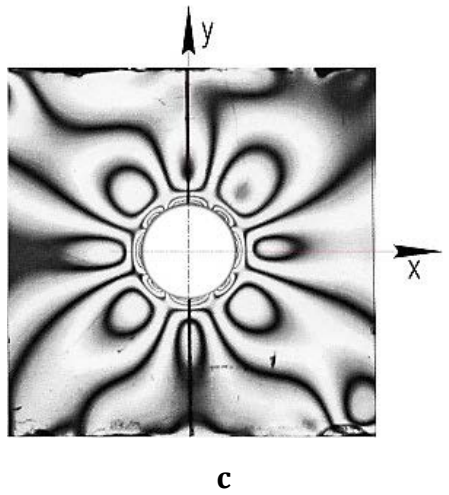

Fig. 9. The method of loading the model with a pure shift:

$\mathbf{a}$ - is the scheme of loading of the plate-model by a pure shear;

b - scheme for determining the moment of torsion of the neck;

c - is the pure shear photogram obtained in the polarized light of a mercury lamp;

$P_{01}$ - shear force; $P_{\text {comp }}$ and $P_{\text {stretch }}$ - compressive and tensile forces of the model, respectively;

$D_{s h}$ and $D_{c s h}$ - the diameters of the connecting rod and the root collar, respectively; $R_{c r}$ - crank radius

The method of loading the model with a pure shift and the photogram of pure shear obtained in the polarized light of a mercury lamp is shown in Fig. 9. After loading the model with computed forces controlled by optical disk dynamometers 1 and 2, we photographed by a digital camera (Fig. 8, 9).

\section{Results and discussions}

From the photograms, we cut out areas in the form of a square, whose sides are located much closer to the holes model, which allows you to build well-visible diagrams of contour stresses. On the holes contour there are four zero points, which serve as the starting point for the order of the bands. They are the boundary of the change in the sign of the stresses from compression to stretching along the contour of the hole. The torsion torque causes on the holes contour the deformation of the pure shear, which creates a sign-alternating stress state stretching $\sigma_{\text {stretch }}$ and compression $\sigma_{\text {comp }}$ successively through $90^{\circ}$ between zero points on the holes contour (Fig. 10).

The photogram obtained by loading a flat model with a pure shear, while transferring its image to the crankpin, is turned counterclockwise by an angle of $45^{\circ}$ and, accordingly, the diagram on the holes contour is oriented with respect to the curve of the contour stresses of stretching from the force from the bending (Fig. 11). Circuits of contour normal stresses at the scale of the bands, built on a quarter of a circle, make it possible to establish separately the maximum stresses from stretching and from shear. Based on these separate diagrams 1 and 2, a total diagram of the normal contour stresses 3 was created (Fig. 12), which indicates that the largest tensile stresses on the holes contour occur in zones located approximately at an angle of $30 \div 40^{\circ}$ to a plane perpendicular to the direction of stretching forces. On the flat models of optically sensitive material, we carried out studies of the stress state 
in the calculated zones of the crankshaft. There searches made it possible to establish the qualitative and quantitative composition of the stresses on the holes contour of the oil channel against deformation of the bending and torsion of its connecting rod. The maximum stresses on the holes contour reach approximately 7 bands at a given load, which is greater than the bending stresses on the crankpin fillet.

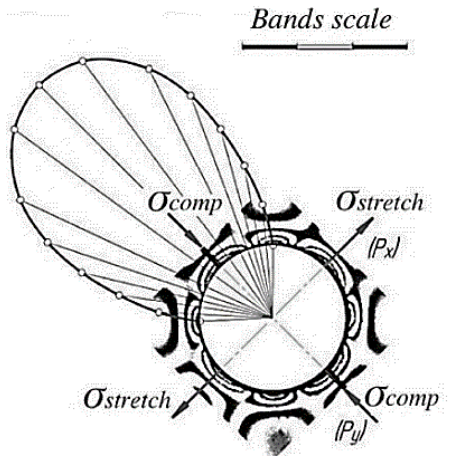

Fig. 10. Diagrams of stretching stresses $\sigma_{\text {stretch }}$ in the bands on the hole contour on the periphery of the crankpin, caused by deformations the shear from torsion

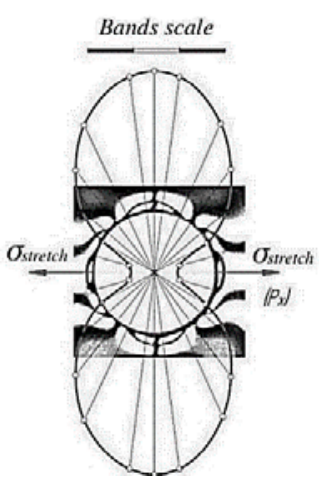

Fig. 11. Diagrams of stretching stresses $\sigma_{\text {stretch }}$ in the bands on the hole contour on the periphery of the crankpin, caused by deformations: the stretching from bending

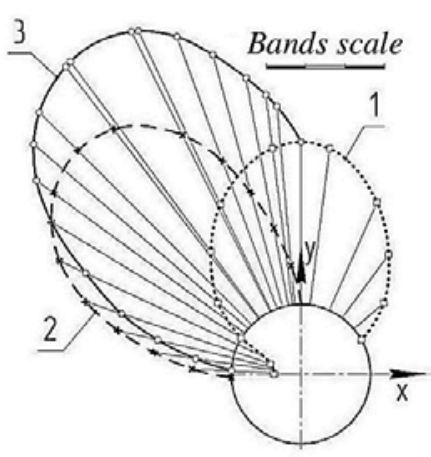

Fig. 12. Diagrams of contour stresses: 1- from bending; 2 - shear; 3 - total diagram of contour stresses in bands per quarter of the hole circle

Additional studies of stresses in the transition fillets from torsional deformations will make it possible to compare the total stresses in the investigated zones under adequate loading conditions.

Observing the similarity criteria, the results can be transferred to the full-scale crankshaft.

\section{References}

1. A.S. Koshelenko, G.G. Poznyak, Theoretical bases and practice of photomechanics in mechanical engineering, Izdatel'skiy dom "Granitsa", 296 p. (2004)

2. A.S. Koshelenko, I.S. Babenkov, O.V. Zhed, Investigation of the stressed state of the crankshaft of the engine by the method of photomechanics, Gruzovik \&, №12, pp. 2124 (2000)

3. A.S. Koshelenko, O.V. Zhed, Polarization-optical model of the crankshaft, Vestnik of the Peoples Friendship University of Russia, Engineering research, №1, pp. 69-73 (2003)

4. V.A. Rogov, A.S. Koshelenko, O.V. Zhed, Investigation by the photomechanics method of the intensity of stresses on the contour of the hole of the oil channel of the crankshaft necks from the workings of the ICE, Gruzovik, №11, pp. 15-19 (2015)

5. A.S. Koshelenko, O.V. Zhed, Investigation on optical plane models of crankshaft stresses from bending and torsion at design points, Tekhnologiya mashinostroyeniya, №10, pp. 52-57 (2016) 